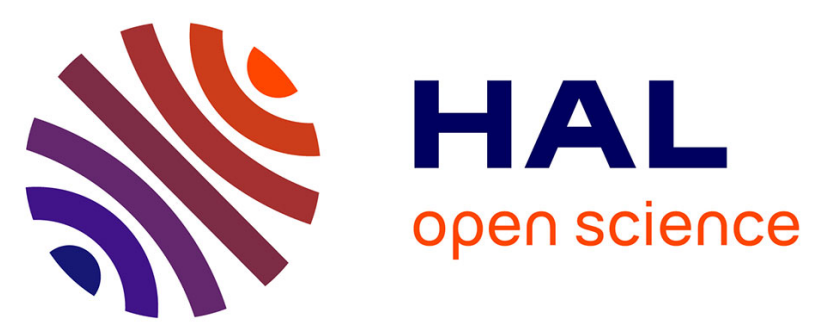

\title{
Étude de la production verbale orale chez les patients parkinsoniens : corrélations entre activité neuronale de structures sous-thalamiques et dénomination d'images
}

Jerome Coste, Cyril Perret, Jean-Jacques Lemaire

\section{- To cite this version:}

Jerome Coste, Cyril Perret, Jean-Jacques Lemaire. Étude de la production verbale orale chez les patients parkinsoniens : corrélations entre activité neuronale de structures sous-thalamiques et dénomination d'images. Réunion de la Société de Neurochirurgie de Langue Française, Société de Neurochirurgie de Langue Française (SNCLF), Dec 2010, Paris, France. pp.546, 10.1016/j.neuchi.2010.10.068 • hal01561337

\section{HAL Id: hal-01561337 \\ https://hal.science/hal-01561337}

Submitted on 8 Dec 2017

HAL is a multi-disciplinary open access archive for the deposit and dissemination of scientific research documents, whether they are published or not. The documents may come from teaching and research institutions in France or abroad, or from public or private research centers.
L'archive ouverte pluridisciplinaire HAL, est destinée au dépôt et à la diffusion de documents scientifiques de niveau recherche, publiés ou non, émanant des établissements d'enseignement et de recherche français ou étrangers, des laboratoires publics ou privés. 


\title{
Étude de la production verbale orale chez les patients parkinsoniens : corrélations entre activité neuronale de structures sous-thalamiques et dénomination d'images
}

\author{
Coste Jérôme ${ }^{1}$, Perret Cyril², Lemaire Jean-Jacques ${ }^{1}$
}

1. Service de neurochirurgie A, université d'Auvergne, UFR médecine, IGCNC, hôpital GMontpied, CHU Clermont-Ferrand, 63000 Clermont-Ferrand, France ;

2. Université de Neuchâtel, NeuroPsychoLinguistic Group, FLSH, ISLL, Neuchâtel 2000, Suisse.

Introduction. La production verbale orale (PVO) implique notamment les ganglions de la base. Deux boucles d'encodage sont proposées : phonologique via le noyau caudé, le pallidum interne et le thalamus ventro-antérieur, et phonétique articulatoire via le putamen, le pallidum interne et le thalamus ventro-latéral. Nous avons émis l'hypothèse que l'activité neuronale de structures ventro-thalamiques et sousthalamiques était modifiée lors de la PVO, en intra opératoire chez des patients parkinsoniens.

Patients et méthode. Huit patients droitiers (quatre hommes, $60 \pm 8$ ans) souffrant d'une maladie de Parkinson sévère depuis $9 \pm 3$ ans ont été implantés bilatéralement dans la région du noyau sous-thalamique (NST) sous anesthésie locale. Les trajectoires étaient déterminées après segmentation manuelle de structures sousthalamiques sur une séquence IRM d'inversion-récupération: NST, zona incerta, substance noire, noyau rouge, noyau de l'anse lenticulaire et champs de Forel. La PVO a été étudiée pendant les enregistrements électrophysiologiques réalisés chaque millimètre sur une distance de $10 \mathrm{~mm}$, simultanément sur deux trajectoires. Trente trois images correspondant à des mots monosyllabiques et monomorphémiques étaient présentées dans trois conditions :

- repos, sans stimulation (contrôle) ;

- le patient pensait au nom de l'image sans le prononcer $(\mathrm{nP})$;

- le patient devait prononcer le nom, et dans ce cas la période de préparation (préP) et celle d'exécution (execP) ont été distinguées.

Nous avons calculé la fréquence moyenne des neurones (FMN, Hz). Les données ont été étudiées avec un modèle mixte de régression linéaire (variables : patients, régions anatomiques et activité électrophysiologique). 
Résultats ou cas rapporté. 1248 sur 1760 enregistrements étaient exploitables. La FMN était supérieure en condition execP que dans les autres conditions (ContrôleexecP, $\mathrm{p}=0,05$; $\mathrm{nP}$-execP, $\mathrm{p}=0,02$; pré $\mathrm{P}$-execP, $\mathrm{p}=0,004)$, cela exclusivement dans le NST (Contrôle-execP, $\mathrm{p}=0,02$; $\mathrm{nP}$-execP, $\mathrm{p}=0,002$; préP-execP, $\mathrm{p}=0,005)$.

Conclusion. Le NST semble avoir une activité augmentée pendant la PVO. Ces résultats doivent être interprétés prudemment pour cet échantillon de huit patients parkinsoniens droitiers. 